\title{
Diphallia: a case report of a rare anomaly evaluated by magnetic resonance imaging
}

\author{
Márcio Luís Duarte ${ }^{1,2 \odot}$, Lucas Ribeiro dos Santos ${ }^{2,3 \odot}$, \\ André de Queiroz Pereira da Silva ${ }^{1,4}$, Marcelo de Queiroz Pereira da Silva ${ }^{1 \odot}$ \\ ${ }^{1}$ WEBIMAGEM Telerradiologia, São Paulo, São Paulo, Brazil; ${ }^{2}$ Master's degree in Evidence-Based Health UNIFESP, São Paulo, São \\ Paulo, Brazil; ${ }^{3}$ Faculdade de Ciências Médicas de Santos, Santos, São Paulo, Brazil; ${ }^{4}$ Hospital São Rafael, Imperatriz, Maranhão, Brazil.
}

\begin{abstract}
Background. Diphallus, also known as penile duplication, is a rare malformation, seen once in every 5 to 6 million births. Newborns showing this condition present higher mortality rates due to malformations and infections. The underlying etiology of this malformation is uncertain, but it is thought to be associated with trauma, drug use, or infections that may affect fetal the mesoderm between the $23^{\text {rd }}$ and $25^{\text {th }}$ day of pregnancy. Our objective is to describe this rare malformation - diphallus - through magnetic resonance imaging, as well as additional findings.
\end{abstract}

Case. A Three-month-old male patient with a 33-week ultrasound demonstrating genital malformation presented to our clinic. At birth, the physical examination revealed diphallia and imperforated anus. Surgical procedures were carried out right after birth to correct the anus malformation. The child did not present any alteration in skin color, and no signs of pain were shown in the abdomen, pelvis, and penises palpations. Urination was observed only through the right penis. Magnetic resonance imaging (MRI) showed two penile structures, each one presenting developed with corpus cavernosum. The penis located on the right showed a complete urethral path in the corpus spongiosum to the vesical floor while the penis located on the left was bigger and did not present a urethral path.

Conclusions. Penile duplication is a rare condition that is often, associated with other malformations, especially anorectal. To fully understand the extension of congenital anomalies and to determine the optimal surgical approach, MRI yields detailed imaging of the entire pelvic region, providing a thorough anatomical frame of reference, and should be routinely incorporated into presurgical evaluation.

Key words: infant, newborn, urogenital abnormalities, anorectal malformations, magnetic resonance imaging.

Duplication of the penis, also referred to as diphallia, is a rare congenital anomaly, with an estimated incidence of 1:5 million live births; ${ }^{1}$ over one hundred cases have been reported since the first documentation by Wecker in 1609. ${ }^{2}$

Cases of diphallia differ greatly from one another, varying from double glans with a common shaft, to complete duplication of the phallus (which is even more infrequent). 3,4

$\bowtie$ Márcio Luís Duarte

marcioluisduarte@gmail.com

Received 29th July 2020, revised 11th November 2020, 3rd December 2020, accepted 9th December 2020.
Other anomalies may coexist, such as the ectopic scrotum, hypospadias, and double bladder. ${ }^{5}$ Gastrointestinal malformations, presenting imperforate anus, colon duplication, and vertebral deformities can often occur with diphallia. ${ }^{5}$ Newborns with this condition have an increased risk of death due to malformations and the associated infections. ${ }^{6}$

Diphallia can be classified as true diphallia or bifid phallus, both of which can be further classified as complete or partial duplication. In complete penile duplication, each penis presents two corpus cavernosum and one corpus spongiosum; in partial diphallia, one of them presents rudimentary structures., 
Bifid phallus, which is responsible for only onethird of the reported cases of diphallia, presents only one corpus cavernosum in each penis, and according to the location of separation, it can be classified as complete when it occurs at the base of the shaft, or partial when it is located as the glans. ${ }^{7,8}$ The bifid phallus is responsible for only one-third of the reported cases of diphallia. ${ }^{7,8}$ Anatomical differentiation may be difficult, and subsidiary diagnostic techniques are most often necessary to plan the therapeutic approach.

Herein, we report a case of a partial true diphallia, correlating with magnetic resonance imaging (MRI) findings. Informed consent was obtained from the patient's parents for the publication of this case report.

\section{Case Report}

A 3-month-old male patient presented to the pediatrics department, with a previous 33-weeks gestational ultrasound demonstrating genital malformation, with possible diphallia, a precise diagnosis could not be reached due to fetus position. At birth, the patient presented with diphallia with two testicles, one in each hemi-scrotum (Fig. 1) and imperforate anus. The mother denied the use of any medications,
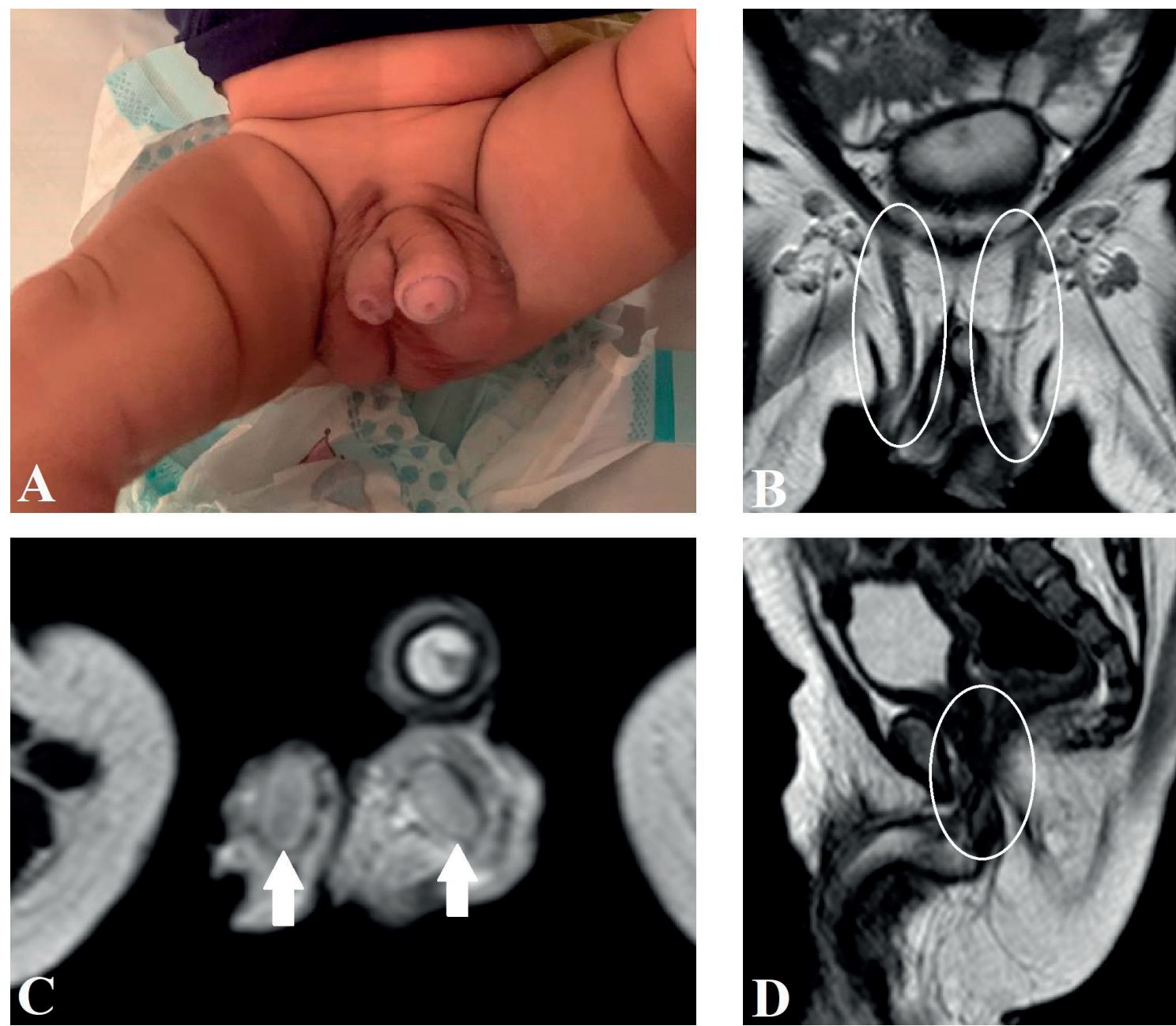

Fig. 1. A: A visual demonstrating two penises - diphallia. B: The MRI in the coronal section in the T2 sequence showing normal spermatic cords (white circles). C:MRI in the axial section in the T2 sequence showing normal testicles (white arrows). D: MRI in the sagittal section in the T2 sequence, the rectum appears to join in the urethra - a defect of the urogenital sinus (white circle). 
alcohol nor drugs during pregnancy. At birth, the patient underwent surgery to correct the imperforate anus. At the consultation, he presented with 2 penises, but only through the right penis was urine outflow observed. The remainder of the physical examination was unremarkable, as anal imperforation had already been corrected.

The patient underwent MRI (Fig. 2), which demonstrated two penile structures. The penis located on the right contained a complete urethral path, through the corpus spongiosum, connecting to the bladder; the penis on the left was larger but did not have a complete urethral path, presenting with an incomplete rudimentary membranous and prostatic urethra. MRI also showed anorectal atresia with a fistulous path, $2 \mathrm{~mm}$ thick, directing to the prostatic/membranous urethra on the right. On MRI two preserved, well located, testicles were observed. After the MRI, the patient has been prepared for penile surgery.

\section{Discussion}

Diphallia is a rare condition, which may be complicated by other congenital anomalies. The cloacal-like (urethrorectal fistula) associated malformation presented by our patient has been previously reported in the literature, being repaired by simple excision. ${ }^{8}$

The underlying etiology of this malformation is uncertain, but it might be associated with trauma, drug use, or infections that may affect the fetal mesoderm between the $23^{\text {rd }}$ and $25^{\text {th }}$ day of pregnancy. ${ }^{7,910}$ It was found that the karyotype in cases of diphallia is normal, except for the case described by Karna and Kapur, associated with a translocation between $46, \mathrm{XY}$, $\mathrm{t}(1: 14)\left(\mathrm{p} 36.3 ; \mathrm{q} 24.3 .^{11}\right.$

Compared to bifid phallus, diphallia usually presents with more complex associated malformations, such as other genitourinary malformations (bladder and urethral duplication, vesical exstrophy, renal abnormalities, and bifid scrotum), as well as anorectal malformations (bowel duplications, fistulas, and anal atresia), and vertebral anomalies. ${ }^{8,12}$

True diphallia rarely occurs in isolation and usually presents with other anomalies, such as hypospadias, epispadias, and ectopic urethra. ${ }^{4}$ The hypoplastic or blind-ended urethra, as seen in our patient. that presents with only one complete and well-developed urethra can also occur.

Penile duplication, as well as associated anomalies, may urge multiple procedures, at different points of time, to achieve full functional and aesthetic conditions. It is fundamental to depict urethral pathways, and which (if one or both) present urine outflow; in most cases, the conventional urodynamic study can determine such characteristics, as well as other renal tract malformations. ${ }^{4}$ However, MRI can further depict other minor associated anomalies, as well as help to decide the surgical approach, as it permits evaluation of penile structures; in fact, comprehensive studies comparing MRI to other imaging techniques have already demonstrated its superiority, yielding greater accuracy. ${ }^{14,15}$

Also, to determine which penis has the full functional capacity, the presence of two corpus cavernosum and one corpus spongiosum is decisive, as well as spermatic cords, as MRI demonstrated in our patient; such structures will determine which penis will be excised. In more complex cases, where there is only one corpus cavernosum in each penis, the joining of two corporal bodies with penile reconstruction has been reported. ${ }^{4,6,10,16,17}$

Every patient with diphallia needs to be carefully examined for the assessment of associated malformations. ${ }^{10}$ The great variability of the anatomy and the presentation in cases of diphallus result in different strategies for the management of malformation. ${ }^{4,10}$ Surgical correction is individualized to achieve adequate urinary continence and erection with adequate aesthetics. ${ }^{4,6,9}$ 

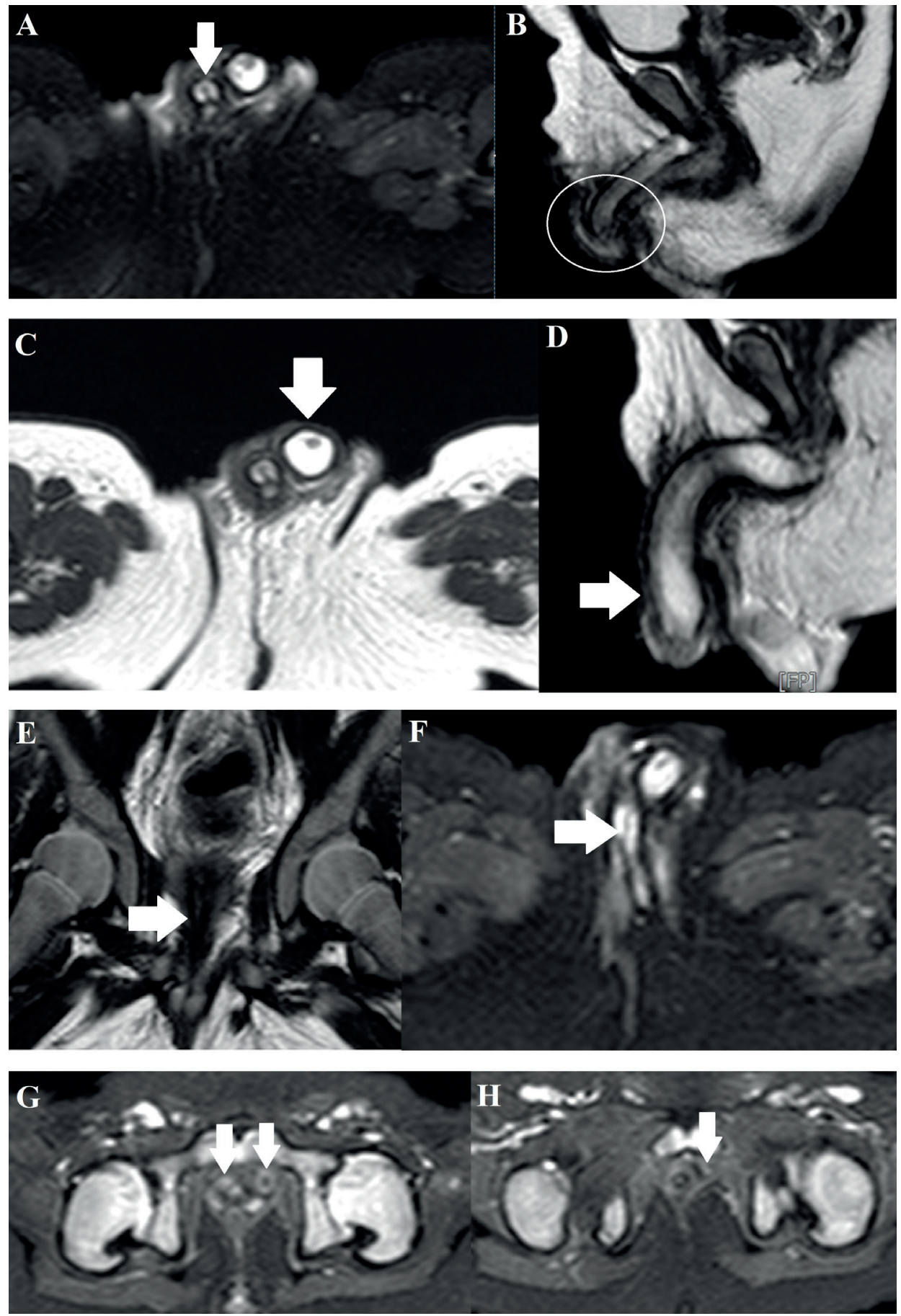

Fig. 2. A: MRI in the axial section in the T2 FAT SAT sequence. B: MRI in the sagittal section in the T2 sequence showing the appearance of the urethra of the right penis (white arrow and circle). C: MRI in the axial view and $D$ : in the sagittal view in the T2 sequence showing a single mass of the corpus cavernosum in the left penis (white arrows). E: MRI in the coronal section in the T2 sequence. F: MRI in the axial section in the T2 FAT SAT sequence showing the right urethra opening into the corpus spongiosum (white arrows). G: MRI in the axial section in T2 FAT SAT sequence showing the two urethrae (white arrows). H: MRI in the axial section in the T2 FAT SAT sequence showing the left urethra ending in a blind bottom (white arrow). 
Penile duplication is a rare condition that is often associated with other malformations, especially anorectal. To fully understand the extension of congenital anomalies and to determine the optimal surgical approach, MRI yields detailed imaging of the entire pelvic region, providing a thorough anatomical frame of reference, and should be routinely incorporated into presurgical evaluation.

\section{Acknowledgment}

To Ms. Roberta Neves Gago Rodrigues for the translation adjustments and spelling correction.

\section{Author contribution}

The authors confirm contribution to the paper as follows: study conception and design: MLD, LRDS, MDQPDS; data collection: MLD, LRDS; analysis and interpretation of results: MLD, LRDS, ADQPDS; draft manuscript preparation: MLD, LRDS, ADQPDS, MDQPDS.All authors reviewed the results and approved the final version of the manuscript.

\section{Conflict of interest}

The authors declare that there is no conflict of interest.

\section{REFERENCES}

1. Torres-Medina E, Sanchez-Puente JC, AragonTovar A. Diphallia, report of one case and review of literature. Rev Mex Urol 2009; 69: 32-35.

2. Neugebauer FL. 37 Fälle von Verdoppelung der äusseren Geschlechtsteile. Monatschr Geburtsh Gynäk 1898; 7: 645-648.

3. Sharma KK, Jain R, Jain SK, Purohit A. Concealed diphallus: a case report and review of the literature. J Indian Assoc Pediatr Surg 2000; 5: 18-21.

4. Karagözlü Akgül A, Uçar M, Çelik F, Kırıştıoğlu İ, Kılıç N. Complete penile duplication with structurally normal penises: a case report. Balkan Med J 2018; 35: 340-343.
5. Carvalho AP, Ramires R, Soares J, Carvalho LF, Filinto M. Surgical treatment of complete penile duplication. Actas Urol Esp 2008; 32: 941-944.

6. Bakheet MA, Refaei M. Penile duplication and two anal openings; report of a very rare case. Iran J Pediatr 2012; 22: 133-136.

7. Frolov A, Tan Y, Rana MW, Martin JR 3rd. A rare case of human diphallia associated with hypospadias. Case Rep Urol 2018; 2018: 8293036.

8. Gyftopoulos K, Wolffenbuttel KP, Nijman RJ. Clinical and embryologic aspects of penile duplication and associated anomalies. Urology 2002; 60: 675-679.

9. Mukunda R, Bendre PS, Redkar RG, Hambarde S. Diphallus with anorectal malformation-case report. J Pediatr Surg 2010; 45: 632-634.

10. Mirshemirani A, Roshanzamir F, Shayeghi S, Mohajerzadeh L, Hasas-Yeganeh S. Diphallus with imperforate anus and complete duplication of rectosigmoid colon and lower urinary tract. Iran J Pediatr 2010; 20: 229-232.

11. Karna P, Kapur S. Diphallus and associated anomalies with balanced autosomal chromosomal translocation. Clin Genet 1994; 46: 209-211.

12. Karaca I, Turk E, Ucan AB, Yayla D, Itirli G, Ercal D. Surgical management of complete penile duplication accompanied by multiple anomalies. Can Urol Assoc J 2014; 8: E741-E743.

13. Muramatsu M, Shishido $S$, Nihei $H$, et al. Urinary reconstruction in vertebral, anorectal, cardiac, trachea-esophageal, renal abnormalities and limb defects association with chronic renal failure and penile duplication. Int J Urol 2015; 22: 125-127.

14. Zhan Y, Wang J, Guo WL. Comparative effectiveness of imaging modalities for preoperative assessment of anorectal malformation in the pediatric population. J Pediatr Surg 2019; 54: 2550-2553.

15. Thomeer MG, Devos A, Lequin M, et al. High resolution MRI for preoperative work-up of neonates with an anorectal malformation: a direct comparison with distal pressure colostography/fistulography. Eur Radiol 2015; 25: 3472-3479.

16. Corrêa Leite MT, Fachin CG, de Albuquerque Maranhão RF, Francisco Shida ME, Martins JL. Penile duplication without removal of corporal tissue: step by step of an excellent cosmetic result. J Pediatr Urol 2014; 10: 567-570.

17. Tirtayasa PM, Prasetyo RB, Rodjani A. Diphallia with associated anomalies: a case report and literature review. Case Rep Urol 2013; 2013: 192960. 